\title{
Computer vision feature recognition method based on Improved Wavelet arithmetic
}

\author{
Liu Lili \\ Zaozhuang Vocational College of Science and Technology,Zaozhuang, Shandong 277599,China \\ zztzliulili@163.com
}

Key words: feature extraction; wavelet moment invariants; binary wavelet

\begin{abstract}
: in the process of studying on computer vision feature recognition method, with the current algorithm for feature recognition, the amount of calculation is large, and noise is strong, recognition rate is low. For this, a computer vision feature recognition method based on Improved Wavelet arithmetic is put forward. Firstly, spline binary wavelet decomposition is fused in the method to extract edge feature from collected images, and then wavelet moment invariants of the processed image is calculated and acted as the characteristic quantity of computer vision, so as to complete the accurate identification of the characteristics of computer vision. Characteristic quantity extracted through this method can not only solve the problem of uneven illumination, illumination variation and noise interference, also translation, rotation and zoom. Simulation results show that accuracy of computer vision feature recognition based on improved wavelet algorithm is great, and effect is ideal.
\end{abstract}

\section{Introduction}

The level of computer science and technology continue to be improved, the application scope of pattern recognition and detection based on digital image is more and wider (1.2.3). During the process of pattern recognition and detection with digital images, recognition of visual feature is the premise of ensuring success of identification and detection(4.5.6). But most of the visual feature recognition methods have limitations, computer vision feature recognition technology is the effective way to solve this problem, and attracts the attention of a lot of experts and scholars (7.8.9).

At present, main computer vision feature recognition methods including the method based on triangular fuzzy number algorithm, neural network algorithm, and double chain genetic algorithm method. Among them, the most commonly used is the computer vision recognition method based on triangular fuzzy number algorithm, but the problem caused by the above algorithm, like the large amount of calculation, strong noise, low recognition rate.

In view of the above problems, a computer vision feature recognition method based on Improved Wavelet arithmetic is proposed. This identification method has the advantages of high accuracy, and effect is ideal.

\section{2 computer vision recognition principle}

Computer vision feature recognition is mainly based on the characteristics of image recognition system, the acquired image feature signal is filtered by a group of band pass filter of different scale, the characteristic signal is decomposed into different frequency bands for analyzing and processing, the frequency band is divided into multiple levels, and the high frequency part of the image features are further decomposed, and according to the characteristics to choose corresponding frequency band adaptively, which can be matched with the image feature signal frequency spectrum, thus completing the computer vision recognition. The specific steps are as follows:

(1) During the feature recognition process of computer vision, firstly, the digital RGB image $S$ is decomposed by $R, G, B$ to obtain $S r, s g, s b$ three images. Then each image separately is decomposed by two layer wavelet packet, to extract signal feature of 16 frequency components from low frequency to high frequency in second layers. 
(2) During the feature recognition process of computer vision, the decomposition coefficients of wavelet packet is reconstructed to extract feature signals of each frequency range, reconstruction feature signal of $x r 20$ is expressed by $S r 20$, reconstruction feature signal of $\mathrm{xr} 21$ is expressed by $\operatorname{Sr} 21$, the other by analogy.

(3) During the feature recognition process of computer vision, the total energy of feature signals of each frequency is calculated. The total energy of feature signal corresponding to $\operatorname{sr} 2 j(j=0,1, \ldots 15)$ is $\operatorname{Er} 2 j(j=0,1, \ldots 15)$, there are:

$$
\operatorname{Er} 2 j=\int|\operatorname{Sr} 2 j(t)| * * 2 * d t=\sum|x r j k| * * 2
$$

Among them, $\operatorname{xrjk}(j=0,1, \ldots, 15, k=1,2, \ldots, n)$ is amplitude of discrete points of reconstruction feature signals.

(4) During the feature recognition process of computer vision, due to each band signal energy of different image is various, so the energy is regarded as an element to construct a feature vector, the feature vector $T$ is constructed as follows:

$$
\operatorname{Tr}=[\operatorname{Er} 20, \operatorname{Er} 21, \operatorname{Er} 22, \operatorname{Er} 23, \operatorname{Er} 215]
$$

(5) During the feature recognition process of computer vision, the image characteristic recognition vector obtained from the color feature vector and wavelet feature vector of image is:

$$
m(k)=\left[c i, \operatorname{Tr}^{\prime} T g^{\prime} T b^{\prime \prime}\right]
$$

\section{Principle of computer vision recognition optimization method}

\section{1 extraction of image edge feature}

During the optimizing process of computer vision feature recognition, assuming $m+1$ order (s) cardinal B spline function can be defined as:

$$
N_{m+1}(t)=N_{1}(t) * N_{m}(t)=\int_{0}^{1} N_{m}(t-u) d u
$$

Among them, $N_{1}(t)$ is the characteristic function of the image frame interval $[0,1]$, * is the convolution operation.

During the optimizing process of computer vision feature recognition, assuming $h, g, \tilde{h}, \tilde{g}$ are finite filters of image recognition, the following equation can be obtained:

$$
\begin{aligned}
& \phi(t)=\tilde{N}_{2 m}(t) \\
& \widehat{h}(\omega)=\sqrt{2}\left(\cos \frac{\omega}{2}\right)^{2 m}
\end{aligned}
$$

During the optimizing process of computer vision feature recognition, assuming $\phi, \varphi, \tilde{\phi}, \tilde{\varphi}$ are limited energy function of image features, frequency domain meet the two scale equation can be obtained by formula:

$$
\begin{aligned}
& \tilde{\varphi}(\omega)=\frac{1}{\sqrt{2}} \tilde{g}(\omega / 2) \tilde{\phi}(\omega / 2) \\
& \hat{\phi}(\omega)=\frac{1}{\sqrt{2}} \tilde{h}(\omega / 2) \tilde{\phi}(\omega / 2) \\
& \varphi(\omega)=\frac{1}{\sqrt{2}} \tilde{h}(\omega / 2) g(\omega / 2)
\end{aligned}
$$

During the optimizing process of computer vision feature recognition, at the same time function of limited energy image features meet the above frequency domain, if $h, g, \tilde{h}, \tilde{g}$ filters satisfied with the formula:

$$
\tilde{h}[\varpi] \tilde{h}(\omega)+g(\varpi) \tilde{g} *(\omega)=2
$$


$\tilde{\psi}$ can be defined as feature information in computer vision feature optimization recognition process.

3.2 implementation of computer vision feature recognition

During the optimizing process of computer vision feature recognition, the equation below is utilized to process weighted sampling for the image:

$$
S q(r i) r i=\sum_{r i-n}^{r i+n} f(x, y) \exp (-i q \theta(x, y)) r(x, y)
$$

The calculation procedure is as follows:

1) During the optimizing process of computer vision feature recognition, the image size is normalized according to requirements. The following equation is used to normalize the image size:

$$
N_{m}(\omega)=\left[\frac{\sin (\omega / 2)}{\omega / 2}\right]
$$

2) During the optimizing process of computer vision feature recognition, the image centroid coordinates is calculated, and the image coordinates is moved to the image centroid. It is expressed by the equation:

$$
\left\{\begin{array}{c}
\tilde{g}(\omega)=\left(1+\sum_{k=1}^{N} a_{k} \cos k \omega\right) \sqrt{2-|\tilde{h}(\omega)|} \\
\tilde{\tilde{g}}(\omega)=\left(1+\sum_{k=1}^{N} a_{k} \cos k \omega\right)^{-1} \sqrt{2-|\tilde{h}(\omega)|^{2}}
\end{array}\right.
$$

3) During the optimizing process of computer vision feature recognition, through calculating to produce $r(x, y)$ and $\theta(x, y)$, which have the same size as image and the same origin of coordinates and the image centroid coordinates.

4) During the optimizing process of computer vision feature recognition, each $S q\left(r_{i}\right) r_{i}$ can be calculated by the following formula:

$$
\left\|F_{m j i}^{\text {wavelet }}\right\|=\left\|\int S_{q}(r) \varphi_{m n}(r) r d r\right\|
$$

In conclusion that accuracy of computer vision feature recognition based on improved wavelet algorithm is great.

\section{4 simulation experiment}

In order to prove the validity of the computer vision feature recognition based on Improved Wavelet arithmetic, there is the need to conduct an experiment. 600 pieces images with noise and 600 pieces images without noise are collected, and Gauss noise of 0.01 variance is added to each image status correspondingly. 500 pieces images are regarded as training samples, the other 700 pieces images are viewed as test samples.

Experiment 1)

With different algorithms to conduct computer vision recognition experiment, the experimental results of recognition rate were compared, the results were shown in table 1.

Table 1 Comparison of recognition rate of different algorithms

\begin{tabular}{ccccc}
\hline Methods & $\begin{array}{c}\text { Training } \\
\text { sample /frame }\end{array}$ & $\begin{array}{c}\text { Testing } \\
\text { sample/ frame }\end{array}$ & $\begin{array}{c}\text { recognition } \\
\text { rate } \\
\text { (noise-free }) \\
/ \%\end{array}$ & $\begin{array}{c}\text { recognition } \\
\text { rate }\end{array}$ \\
$\begin{array}{c}\text { (noise) } \\
/ \%\end{array}$ \\
\hline $\begin{array}{c}\text { traditional } \\
\text { algorithm } \\
\begin{array}{c}\text { improved } \\
\text { algorithm }\end{array}\end{array}$ & 500 & 700 & 85 & 32 \\
\hline
\end{tabular}

It can be seen from table 1, the accuracy of improved algorithm is higher than traditional 
algorithm in the feature recognition, it is because spline binary wavelet decomposition is fused in the method to extract edge feature from collected images, and then wavelet moment invariants of the processed image is calculated and acted as the characteristic quantity of computer vision, so as to complete the accurate identification of the characteristics of computer vision.

\section{Experiment 2)}

Different algorithms were utilized for computer vision feature recognition, and the different recognition effect were compared. The results shown in figure 1:

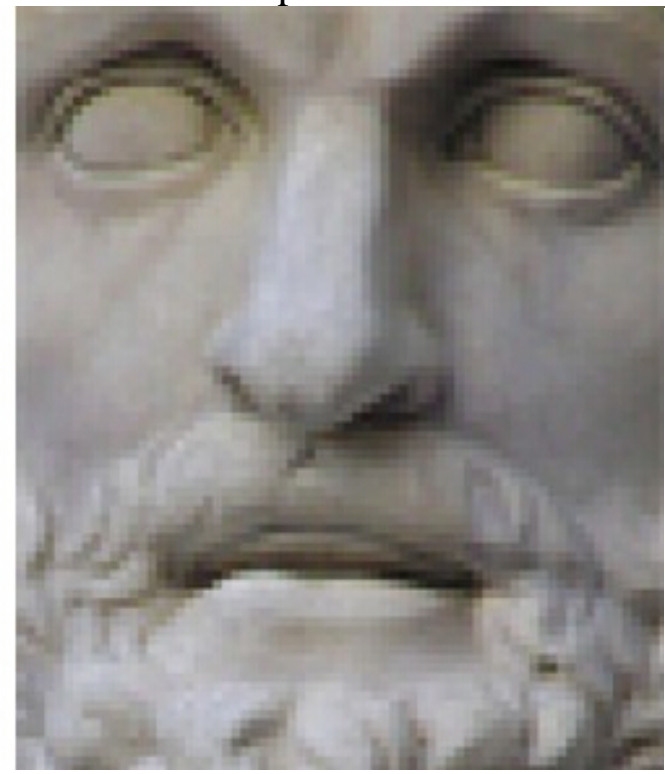

(a)traditional algorithm

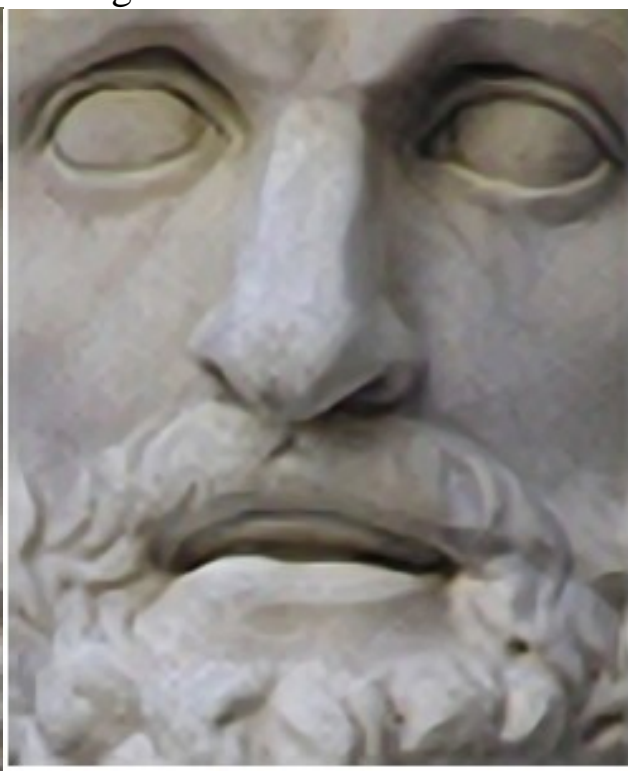

(b)improved algorithm

Fig. 1 Comparison of effect of computer vision recognition with different algorithms As can be seen from Figure 1 that, the recognition effect of the proposed algorithm is better than the traditional algorithm, because the improved algorithm utilizes wavelet moment invariant and the ability to grasp the details of the image, thus, in the recognition of computer vision feature, visual effect is good.

\section{Conclusions}

In view of the current algorithm for feature recognition, the amount of calculation is large, and noise is strong, recognition rate is low. For this, a computer vision feature recognition method based on Improved Wavelet arithmetic is put forward. Firstly, spline binary wavelet decomposition is fused in the method to extract edge feature from collected images, and then wavelet moment invariants of the processed image is calculated and acted as the characteristic quantity of computer vision, so as to complete the accurate identification of the characteristics of computer vision. Characteristic quantity extracted through this method can not only solve the problem of uneven illumination, illumination variation and noise interference, also translation, rotation and zoom. Simulation results show that accuracy of computer vision feature recognition based on improved wavelet algorithm is great, and effect is ideal.

\section{References}

[1] Shang Shuling. Logistics Sorting Violence Behavior Recognition Based on Computer Vision [J]. Computer Simulation. 2013.12:430-433.

[2] Zhao Haiyan, Niu Wenliang, Liu Kun. Research and Simulation on the Visual Identification Method for Bad Point of Precision Metal Welding [J]. Computer simulation.2014.8:204-208.

[3] Jiang Daewoo. Study on clustering image segmentation algorithm based on improved K-means

[J]. Journal of Harbin University of Commerce: Natural Science Edition.2013.5:575-578.

[4] Ilham Jarl Maimaiti. Human Face Recognition Based on Improved PCA and LDA Fusion 
Algorithm [J]. Computer simulation.2013.1:415-418.

[5] Guo Tingting, Fan Tiesheng. Study of face recognition method based on improved local two value model [J]. Digital technology and application.2013.12:71-72.

[6] Ma Fuyang, Xie Rui. The human pulse signal filtering algorithm research based on improved threshold wavelet decomposition and empirical mode decomposition. 2014.2:37-39. [J]. electronic products in the world

[7] Kang Shouqiang, Li Zhuqiang, Yang Guangxue, Wang Yujing. The application of Wavelet packet-locally linear embedding algorithm in fault level identification of rolling bearing [J]. Chinese Journal of scientific instrument.2014.3:614-619.

[8] Huang Liwen, Wang Xin, Wang Tao, Pang Ke, Cuan Wannian. A kind of craniofacial X image retrieval method based on shape features [J]. Journal of Chongqing University of Technology: Natural Science Edition.2013.6:72-75.

[9] Wang Zhengyou. The recognition method of center of gas flow distribution based on image processing [J]. Computer simulation.2013.9:357-360. 